\title{
THE AD VACUUM SYSTEM: CONSTRUCTION AND COMMISSIONING
}

\author{
M. Brouet, J. Hansen, H. Klette, P. M. Strubin, CERN, 1211 Geneva 23, Switzerland
}

\begin{abstract}
CERN has built a new experimental facility, called the Antiproton Decelerator (AD), by transforming two existing machines: the "Antiproton Collector" (AC) and the "Antiproton Accumulator" (AA). To achieve adequate beam performance once the antiproton beam is decelerated to its final value of $0.1 \mathrm{GeV} / \mathrm{c}$, it was necessary to lower the average pressure by nearly two orders of magnitude. For this purpose, a large number of additional pumps were installed and a very careful preparation was applied to a variety of special machine equipment which, in its original construction, was not designed for ultra-high vacuum operation. An important improvement in the outgassing rates was achieved through an extended, mild bake-out of tanks and vacuum vessels containing large amounts of ferrite material and multi-layer thermal insulation.

This paper describes the necessary modifications of the vacuum system and in more detail the various steps taken to obtain the required pressure in the low $10^{-10} \mathrm{mbar}$ range. It will also report on the unexpected difficulties which were encountered by re-using vacuum components that were not initially designed for bake-out.
\end{abstract}

\section{INTRODUCTION}

Following the decision to close the LEAR antiproton program, there was a strong request from the user community to continue certain experiments. The Antiproton Decelerator (AD) project was approved after a careful study of the feasibility of converting the existing Antiproton Collector (AC) into the new facility [1]. In addition, some parts of the LEAR ring, notably the electron cooling system, were recuperated and adapted for AD.

The decision to build this new facility with mostly existing equipment was justified by a lower cost and faster installation time. However, it required the Vacuum Group to apply a number of original solutions in order to meet the design pressure of $510^{-10} \mathrm{mbar}$. It also involved a number of unexpected difficulties, mainly when trying to apply mild bake-out to equipment which was not foreseen to be baked at all.

The first measure was obviously to increase the pumping speed wherever possible, in particular by the addition of about one hundred titanium sublimation pumps. The second measure was to minimize the outgasing rates of selected components, like injection and ejection kickers, RF cavities or stochastic cooling pickups and kickers. This was achieved by performing low temperature bakeout on these components. Finally, installing ion gauges, cold cathode gauges and gas analyzers, not previously available in AC, allowed for better diagnostic.

\section{ASSESSING THE NEEDS}

The goal of the improvement of the vacuum system was to reach an average pressure in the low $10^{-10} \mathrm{mbar}$, with residual gas mainly dominated by hydrogen [2]. The average pressure of the former $\mathrm{AC}$ was in the high $10^{-9}$ mbar range, with a significant amount of water vapor and carbon monoxide in addition to hydrogen. Calculations of the expected pressure profile were carried out in order to identify the most critical parts so as to concentrate the effort on them. Most effort was spent in order to achieve the lowest possible pressure in the regions where the antiproton beam has the highest vertical $\beta$ function. These regions are mainly where special equipment, like kickers and septa are installed.

\section{INCREASING THE PUMPING SPEED}

\subsection{Bending sections}

The pumping speed in the bending sections could be significantly increased by the addition of titanium sublimation pumps at each end of the vacuum chamber of every bending magnet. For this purpose, openings of 100 $\mathrm{mm}$ had to be drilled in the existing vacuum chamber and pumping ports were welded onto the chamber to support the pumps. In order to avoid a manpower-intensive dismounting of the vacuum chamber from the bending magnets, an original tool was developed with the help of an outside company which allowed drilling the holes in situ. A support rod was welded on the vacuum chamber at the center of the piece to be cut out to guide the circular saw. No lubricant was used during the drilling, to avoid any contamination of the vacuum chamber and the opening was de-burred with special care to avoid that metal chips were spread into the vacuum chamber. Welding the pumping ports onto the vacuum chamber and leak testing the welds was all done in situ. In total, 98 sublimation pumps have been added, increasing the total pumping speed for hydrogen by $78^{\prime} 000 \mathrm{l} / \mathrm{s}$ over $70 \%$ of the circumference.

\subsection{Injection, ejection and stochastic cooling kickers}

As there were no additional ports available on these kickers, a tee piece fitted on the entrance flange of the ion pumps was added in order to accommodate two titanium 
sublimation pumps per tank. In addition, some ion pumps were exchanged to larger ones.

\section{4 ВАКE-OUT}

As stated earlier, only parts of the decelerator, which presented excessively high degassing surfaces, were considered for baking. These include the RF cavities, the injection septum magnet, the injection and ejection kickers, the stochastic cooling pick-ups and kickers. However, because these components were not foreseen to be baked at all, temperatures had to be limited to a maximum of $150{ }^{\circ} \mathrm{C}$, even below $100{ }^{\circ} \mathrm{C}$ for the $\mathrm{RF}$ cavities. To compensate for these low temperatures, the bake-out has been extended up to three weeks for some components.

Low-cost, low-temperature heating elements were used where adequate instead of the traditional heating tapes or heating jackets. These heating elements, with a specific heating capacity of $50 \mathrm{~W} / \mathrm{m}$ can be cut to custom lengths, still allowing for direct mains $(230 \mathrm{~V})$ supply. As they are very flexible, they easily follow the sometime difficult shapes of the equipment to be baked. Heat-resistant tape is used to affix them on the surface of the vacuum vessel which is then covered with standard heat-insulating material.

The installed power of the heating elements was calculated to have a limited value, such as to make sure that under no circumstances would the temperature of the vacuum vessel exceed $150{ }^{\circ} \mathrm{C}$. The price to pay for this is a longer rise time up to operating temperature. Also, in addition to the thermocouples required for regulation, a number of redundant thermocouples were installed and connected to a data acquisition system for monitoring and alarm purposes.

\subsection{Bake-out of the RF Cavity}

The RF cavity is a huge device of 2.2 meter in diameter and 2 meter in length, made of aluminium alloy. The flanges, however, are made out of stainless steel. The major worry was to minimize the temperature gradient at the aluminium-to-stainless-steel junctions, which are friction welded. The cavity is pumped by a 20'000 1/s cryopump, switched on after initial pumping with a mechanical pumping station (a set of roughing and turbomolecular pumps).

Initial tests were made in the laboratory by building a hut around the cavity with spare shielding blocks. A $16 \mathrm{~kW}$ air blower was used to provide the heating power, which was able to bring the temperature of the cavity to $60{ }^{\circ} \mathrm{C}$ after 2 days. The cavity was instrumented with thermocouples located in all critical areas and connected to a temperature scanner and logger. The measurements showed that the temperature differences between various parts of the cavity could be kept below $3{ }^{\circ} \mathrm{C}$.

Starting from a pressure of about $10^{-4}$ mbar after initial pumping, the pressure rose into the $10^{-1}$ mbar range as soon as the temperature of the cavity reached $50{ }^{\circ} \mathrm{C}$. From then on, the pressure continuously decreased and reached $10^{-4}$ mbar after 24 hours with an average temperature of the cavity of $60{ }^{\circ} \mathrm{C}$. After cooldown, the pressure was 5 $10^{-8}$ mbar with turbomolecular pumping only. This figure has to be compared with $410^{-7}$ mbar after 8 days of pumping without bake-out.

This first experiment proved that a considerable improvement in the degassing rate of the surface was achievable by applying a mild bake-out to this cavity. However, although quite easy to set up in the laboratory, the heating method was considered to be too space consuming for the accelerator environment. It was therefore decided to order tailor-made heating jackets from industry, with a limited power rating to ensure that under no circumstances would the temperature of any part of the cavity exceed $120{ }^{\circ} \mathrm{C}$. An adequate programming of the temperature regulators insures that the required temperature gradients are satisfied. In addition, a computer monitoring extra temperature probes was programmed to stop the bake-out process should the temperature of one heating element rise above a predefined limit.

The spare cavity was equipped with the heating jackets and baked in the laboratory. Although the overall improvement was comparable to the first cavity, the pressure increase during the initial heating period was much smaller. The origin of this difference in behavior still remains to be studied.

The operational cavity has now been equipped with the jackets and the bake-out in the AD accelerator is foreseen for end of March 1999.

\subsection{Bake-out of the Stochastic Cooling Pick- ups}

The stochastic cooling pick-ups are operated at cryogenic temperatures of some $20 \mathrm{~K}$. The active parts of the pickup are thermally shielded from the vacuum vessel by multiple layers of superinsulation which act as a very large source of gas. Furthermore, the layers of superinsulation were seamed together at their extremities, which prevented the gas trapped between layers to be pumped. As a first step, the welds between layers of superinsulation were cut off in such a way as to allow for the gas to escape. Tests were then made to try and assess the required baking time to achieve the foreseen pressure decrease. When heating was only applied to the outer surface of the vacuum vessel, there was so little thermal transmission through the insulation layers to the inner structure of the pickup assembly that literally months of bake-out would have been required. Furthermore, the pickup electrodes being actively cooled under operation, heat was evacuated via the leads from the electrodes to the housing of the cold heads, the latter being dismantled during bake-out. To overcome this problem, the cold heads are replaced during bake-out by a copper block which is heated and hence transfers thermal power to the inner structure of the pickup assembly. To avoid too strong oxidation of the copper, the volume housing the copper block is pumped out. The atmospheric pressure applied is 
also used to improve the thermal contact between the copper block and the leads to the pickup assembly. With this improvement, the temperature of the pickup electrodes reached $95^{\circ} \mathrm{C}$ within 3 days as shown in Figure 1.

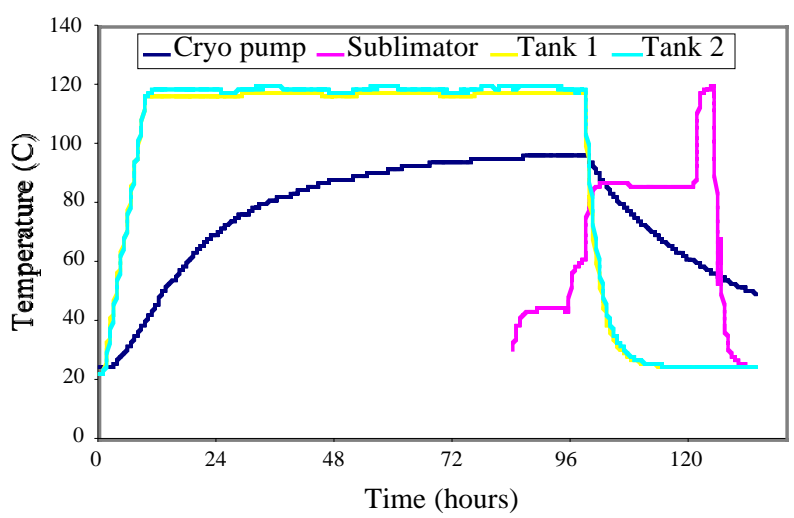

Figure 1: Evolution of temperature during bake-out of stochastic cooling pickup

\section{ADDITIONAL VACUUM INSTRUMENTATION}

The original Antiproton Collector was only equipped with the bare minimum of gauges, the pressure values being mostly evaluated from the current of the ion pumps. A total of 17 Bayard-Alpert gauges were installed all around the ring to allow for pressure measurement in the low 1010 mbar range. These gauges, as well as the cold cathode gauges, are connected to the $\mathrm{AD}$ control system allowing for short- and long-term data logging.

Seven residual gas analyzers were mounted. In addition to allowing for a measurement of the quality of the achieved vacuum, these instruments proved very useful to diagnose a series of very nasty leaks on feedthroughs which were submitted to a pressure of 2 bar of $\mathrm{SF}_{6}$ gas.

\section{CONCLUSIONS AND FUTURE IMPROVEMENTS}

The additional pumping speed obtained with the titanium sublimation pumps and the soft bake-out of the components with the highest degassing rates allowed to reach the required pressure without major modification of the existing equipment.

Figure 2 shows a comparison between the pressures measured in the former $\mathrm{AC}$ compared to pressures in $\mathrm{AD}$, when available.

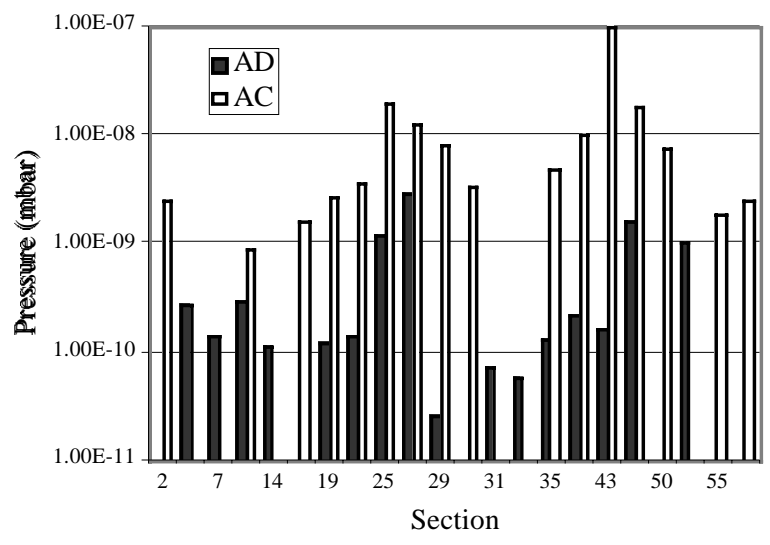

Figure 2: Pressure comparison between $\mathrm{AC}$ and $\mathrm{AD}$

As a further step, it is foreseen to change the NEG cartridge used in the injection septum to a low-temperature one in such a way as to allow its use as both a heating element during bake-out and an active pump. Other studies have started to improve the venting of the vacuum system and to possibly work with a small overpressure of nitrogen during interventions.

\section{REFERENCES}

[1] The Antiproton Decelerator: AD, S. Baird et al., CERN, PAC 1997

[2] Upgrading The AC Vacuum System for the Antiproton Decelerator, M. Brouet and O. Gröbner, CERN Vacuum Technical Note 97-06, April 1997 